\title{
Multilineage-differentiating Stress-enduring (MUSE) Cells in Orthobiologics: Are they the Future?
}

\author{
Eduard Alentorn-Geli ${ }^{1,2,3}$, Patricia Laiz ${ }^{1,2}$, Alfred Ferré-Aniorte ${ }^{1,2}$, Roberto Seijas ${ }^{1,2}$, David \\ Barastegui $^{1,2,3}$, Pedro Álvarez-Díaz ${ }^{1,2,3}$, Xavier Cuscó ${ }^{1,2}$, Cristina Sánchez ${ }^{1,2}$, Luís García ${ }^{1,2}$, \\ Montse García-Balletbó ${ }^{1,2}$, Ramón Cugat ${ }^{1,2,3}$
}

\section{Abstract}

Multilineage-differentiating stress-enduring (MUSE) cells are non-tumorigenic pluripotent stem cells with endogenous reparative properties. These cells have a very powerful ability to adapt to global environment changes and are thus stress-tolerant cells. Interestingly, MUSE cells can differentiate into cells representative of all three germ layers. There has been a number of studies demonstrating its powerful regenerative power in several disorders: type-1 diabetes mellitus, myocardial infarction, stroke, glomerular-related kidney diseases, chronic liver failure, and ischemia-reperfusion lung injury. Recent data have also suggested that MUSE cells have significant repair properties for osteochondral lesions. The present article will review what are MUSE cells and how they work, the application of these cells into different disorders, and the studies upto-date regarding MUSE cells in orthobiologics.

Keywords: Muse cells; stem cells; regenerative; regeneration

\section{Introduction}

The area of regenerative medicine has dramatically evolved in the past decades. The understanding of the mechanisms and advances in their therapeutic properties of stem cells are among the most developed fields in regenerative medicine. The decade of embryonic stem cells in the late 90 s lead to the more recent decade of adult stem cells. Multilineage-differentiating stress-enduring (MUSE) cells are one type of adult stem cells that were discovered accidentally in 2007 by Professor Mari Dezawa [1].

MUSE cells are non-tumorigenic pluripotent stem cells with endogenous reparative properties. These cells mobilize from the bone marrow, dermal fibroblasts, and adipose tissue into the circulating blood when a tissue is severely damaged and replace injured cells to repair and maintain tissues [1]. They have a specific receptor that can respond to damage signals, enabling MUSE cells to preferentially migrate, differentiate and accumulate into the damage tissue. Therefore, they can target multiple tissues and diseases. In fact, these cells execute their reparative effects through paracrine, antiinflammatory, anti-fibrotic, anti-immune, and anti-apoptotic mechanisms. MUSE cells have been shown to replace damaged cells from the heart, liver, kidney, brain, and other organs [1]. More recently, they potential therapeutic properties have been used in osteochondrallesions [2].

The purpose of this article is to review the mechanism of action and therapeutical properties of MUSE cells and the potential role in orthobiologics.
What are Muse Cells and How they Work MUSE cells are one type of mesenchymal stem cells with the ability to differentiate into all three germ layers. They are pluripotent, non-tumorigenic cells that express both the embryonic stem cell marker stage-specific embryonic antigen-3 (SSEA-3) and the mesenchymal cell marker CD105. These cells remain quiescent in the so far identified bone marrow, dermal fibroblasts, and adipose tissue [3]. When significant tissue damage occurs, they become active, detach from their tissues, become pluripotent, and enter the bloodstream without $24 \mathrm{~h}$ after the onset of tissue injury. Then, they home to injured tissues and produce the tissue-specific cells to repair the damage [1]. Its stress-tolerant characteristic allows them to effectively

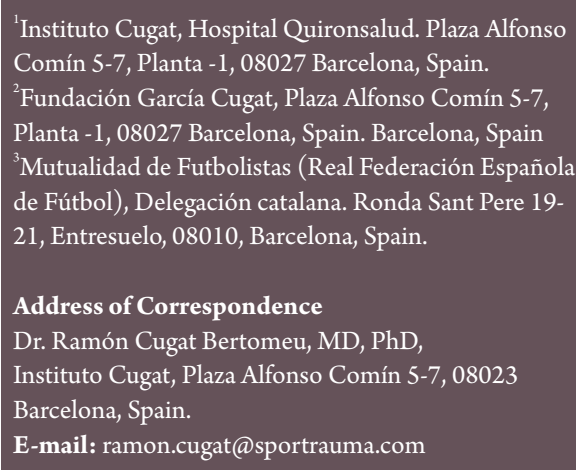

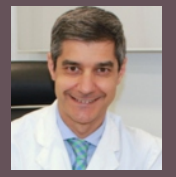

Dr. Eduard Alentorn-Geli

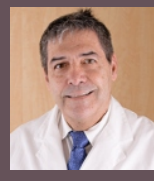

Dr. Xavier Cuscó

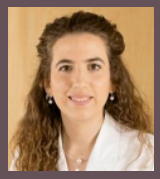

Dr. Patricia Laiz

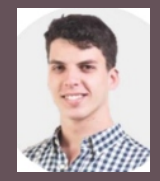

Dr. Alfred FerréAniorte

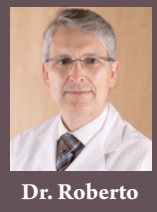

Seijas

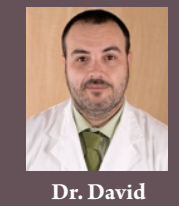

Dr. David Barastegui

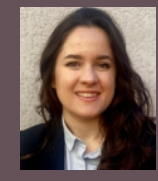

Dr. Cristina Sánchez

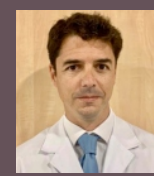

Dr. Luís García

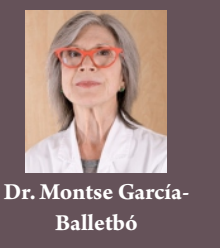

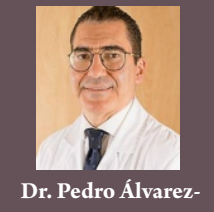

Díaz

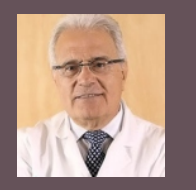

Dr. Ramón Cugat

Submitted Date: 15 September 2021, Review Date: 17 November 2021, Accepted Date: 25 November 2021 \& Published: 31 December 2021

(C) 2021 by Journal of Regenerative Science | Available on www.jrsonweb.com | DOI:10.13107/jrs.2021.v01.i01.023

This is an open access journal, and articles are distributed under the terms of the Creative Commons Attribution-NonCommercial-ShareAlike 4.0 License (https://creativecommons.org/licenses/by-nc-sa/4.0/), which allows others to remix, tweak, and build upon the work non-commercially, as long as appropriate credit is given and the new creations are licensed under the identical terms. 
survive into the hostile environment of tissue damage including the highly aggressive immune response. As mentioned, these cells have specific receptors able to respond to damage signals when tissues are injured. The most important mechanism for detecting damaged tissue and become active for this damage is the sphingosine-1-phosphate (S1P)-S1P receptor 2 (S1PR2) system [4]. The alerting signal S1P attracts MUSE cells via the S1PR2, enabling them to preferentially migrate into the damaged tissue. Interestingly, both endogenous and exogenously administered MUSE cells are able to sense the location of tissue damage and migrate into the needed area. Then, the cells are able to preferentially migrate, differentiate and accumulate into those specific tissues that have been injured, helping in the repair process. MUSE cells survive and remain integrated in the host tissue for an extended period of time, so that their anti-fibrotic, anti-inflammatory, antiapoptotic, and paracrine effects are longlasting $[4,5,6]$. These cells must be collected from the tissue source and then they can be either intravenously administered or placed directly in the tissue repair site.

\section{Therapeutic Properties of Muse Cells in Different Disorders}

One of the therapeutic fields of MUSE cells has been diabetes mellitus [7]. These cells have a significant amount of transforming growth factor-B1 secretion that can downregulate $\mathrm{T}$ lymphocytes and macrophages involved in the auto-immune reaction of type-1 diabetes mellitus. Diabetic NOD mice received either peritoneal $\mathrm{PBD}$ injection (control group) or $1 \times 106$ MUSE cells injection. The experimental group had the blood glucose levels under better control and maintained their body weight, whereas mice in the control group raised blood glucose levels $>500 \mathrm{mg} / \mathrm{dl}$ and diminished body weight abruptly [7].

MUSE cells have also demonstrated that they may improve the prognosis of patients suffering from acute myocardial infarction [8]. Patients with higher concentration of peripheral blood MUSE cells in the acute phase had a more favorable improvement of cardiac function. After intravenous administration, these cells can differentiate into cardiomyocytes and vessels, show paracrine effects, reduce myocardial infarction size by $52 \%$, and produced longlasting improvement of the cardiac function (left ventricle function) and remodeling (attenuates left ventricle remodeling with attenuation of fibrosis) for 6 months $[4,8]$. This is mainly achieved by the S1P-S1PR2 axis in which there is an interaction between the S1P molecule produced by the damaged heart and the S1PR2 located on MUSE cells [4].

Patients suffering for the highly lethal or highly disabling stroke may also benefit from MUSE cells. Despite there are many studies investigating on the regenerative potential of stem cells into ischemic stroke [9], MUSE cells may be among the best performers. Uchida et al. investigated the effects of MUSE cells injection into the peri-infarct areas either at the acute or subacute phase in transient middle cerebral artery occlusion model (large brain infarction model) in rats, and small subcortical infarcts on the corticospinal tract and pure motor paresis model (lacunar infarction model) in mice [10]. The authors observed that MUSE cells survived in the hostile stroke environment and differentiated spontaneously into neurons (60\%) and oligodendrocytes (20\%). The intracerebral injection of MUSE cells could improve motor function at 8-12 weeks in both models $[9,11,12]$. and sensory function in both histological and electrophysiological evaluations [11].

The use of MUSE cells as an experimental therapy for chronic kidney disease has also been investigated [13]. Intravenously injected MUSE cells differentiated into glomerular cells and resulted in improvement in renal function in a murine model of chronic kidney disease. The cells were able to express higher levels of renal markers WT1 and EYA1 compared to non-MUSE cells. Renal function expressed as urine protein-tocreatinine ratio, creatinine clearance, and plasma creatinine was significantly better at 5 weeks compared to vehicle and non-MUSE cells, but these differences were not maintained at 7 weeks.

Nishizuka et al. investigated the role of MUSE cells for liver regeneration [14]. One week after physical partial hepatectomy, MUSE cells were found in the transection line and periportal regions close to the actual injury. At two weeks, MUSE cells began to form bile duct-like structures and at 4 weeks, this population of cells had integrated into the liver and differentiated into cholangiocytes (18\%), hepatocytes (74\%), Kupffer cells (6\%), and sinusoidal endothelial cells (2\%). MUSE cells expressed at 2 days and one week liver progenitor markers such as CK19, deltalike protein, OV-6, and AFP. At 2 weeks, there was no expression of liver progenitor markers but markers of the cells previously indicated: hepatocyte: Hep Par-1, Albumin, alpha-1antitrypsin; cholangiocytes: CK7; sinusoidal endothelial cells: Lyve-1; and Kupffer cells: CD8 [14]. Iseki et al. also demonstrated the liver regeneration capacity of MUSE cells in mouse model liver fibrosis [5].

Yabuki et al. reported on the efficacy of MUSE cells for ischemia-reperfusion lung injury $[15,16]$. The authors provided a rat model in which warm ischemia and subsequent reperfusion with severe pulmonary edema were created. The administration of MUSE cells immediately after reperfusion significantly increased lung oxygenation capacity, compliance, and improved histological damage compared to non-MUSE mesenchymal stem cells [15]. MUSE cells also expressed higher levels of proteins related to anti-inflammation, antiapoptosis, and tissue repair in the lung.

Yamashita et al. described the effects of MUSE intravenous injections in mice model with amiotrophyc lateral sclerosis (ALS). Authors reported a greater migration of MUSE cells into the thoracic and cervical spine in subjects after intravenous injections compared with intratecal injections, probably due to the ability of MUSE cells to migrate and differentiate into apoptotic / damaged cells through S1PR2. Mices were divided into wild type, vehicle groups, mesenchymal stem cells (MSCs) group and MUSE group. At the end of the study, MUSE group showed greater rotarod test, hanging-whire test and muscle strength of lower limbs scores than vehicle and MSCs groups. In hystologics examinations, MUSE group showed a greater motoneuron survivorship at the ventral horn and improved tibialis anterior synapses compared with vehicle and MSCs groups. These results suggests that MUSE intravenous injections could be a future treatment for human patients with ALS [17].

\section{Muse cells in orthobiologics}

There is very limited research on MUSE cells in orthobiologics. Mahmoud et al. conducted a rat animal model study in which they 
investigated the therapeutic potential of MUSE cells in osteochondral lesions [2]. The authors isolated these cells from human bone marrow mesenchymal stem cells and transplanted them into the osteochondral defect of the patellar groove of immunodeficient rats. The 16 rats had a 2 $\mathrm{mm}$ diameter osteochondral defects created using a metallic drill, and then they were unequally distributed into three groups: control group (PBS injection), non-MUSE cells group (intra-articular injection of nonMUSE cells), and MUSE cells group (intraarticular injection of MUSE cells $5 \times$ 104). A macroscopic, histological, and immunostaining analysis for the type of collagen produced was conducted at 4 and 12 weeks after treatment. On the macroscopic examination, the authors found better filling of the defect with smooth cartilage in the MUSE cells group compared to the other two groups. In fact, the control group rats showed osteoarthritic changes in the joint. The MUSE cells group had a significantly higher macroscopic score compared to the other two groups. The former group also had complete repair with a significantly better histologic score compared to the other two groups at 12 weeks. In addition, the cell density of the repair group was significantly higher in the MUSE cells compared to the other two groups. Finally, there was no presence of either type I or II collagen in the repair tissue of the MUSE cells group at 4 or 12 weeks post-operative. This study demonstrated that MUSE cells could be a promising option to repair osteochondral injuries, but the formation of fibrous tissue could not be prevented and the absence of type- 2 collagen indicates that the type of tissue formed was not hyaline cartilage.

An interesting characteristic of MUSE cells is that they can be used through exogenous administration. These cells have an immunomodulatory effect that allows the use of allograft or xenograft cells without a rejection reaction, thus escaping the host immunologic attack $[4,6]$. The study by Mahmoud et al. used MUSE cells as xenografts, which is a clear demonstration of this interesting property.

Toyoda et al. conducted a basic science study were they tried to isolated MUSE cells from the middle-aged to elderly human synovial membrane [18]. The authors isolated cells positives for SSEA-3, a marker of MUSE cells, that expressed NANOG, OCT3/4, and SOX2. Interestingly, cell pellets created from these cells demonstrated chondrogenic potential, as were aggrecan-positive and type2 collagen positive by immunostaining.

\section{Conclusions}

MUSE cells have demonstrated excellent performance for different conditions in the field of regenerative medicine. Despite more than 10 years of research, only very limited studies are available for orthopedics. According to its regenerative potential and availability, MUSE cells could be a feasible option to help in the repair and regeneration tendons, ligaments, menisci, bones, and cartilage injuries. Further investigations are needed in order to confirm the regenerative potential of MUSE cells for the musculoskeletal system.

Declaration of patient consent: The authors certify that they have obtained all appropriate patient consent forms. In the form, the patient has given his consent for his images and other clinical information to be reported in the Journal. The patient understands that his name and initials will not be published, and due efforts will be made to conceal his identity, but anonymity cannot be guaranteed.

Conflicts of Interest: Nil. Source of Support: None.

\section{References}

1. Dezawa M. Muse cells. In: Endogenous Reparative Pluripotent Stem Cells. Japan: Springer; 2018.

2. Mahmoud EE, Kamei N, Shimizu R, Wakao S, Dezawa M, Adachi N, et al. Therapeutic potential of multilineage-differentiating stress-enduring cells for osteochondral repair in a rat model. Stem Cells Int 2017;2017:8154569.

3. Kuroda Y, Kitada M, Wakao S, Nishikawa K, Tanimura Y, Makinoshima $H$, et al. Unique multipotent cells in adult human mesenchymal cell populations. Proc Natl Acad SciU SA 2010;107:8639-43.

4. Yamada Y, Wakao S, Kushida Y, Minatoguchi S, Mikami A, Higashi K, et al. S1P-S1PR2 axis mediates homing of muse cells Into damaged heart for long-lasting tissue repair and functional recovery after acute myocardial infarction. Circ Res 2018;122:1069-83.

5. Iseki M, Kushida Y, Wakao S, Akimoto T, Mizuma M, Motoi F, et al. Muse cells, nontumorigenic pluripotent-like stem cells, have liver regeneration capacity through specific homing and cell replacement in a mouse model of liverfibrosis. Cell Transplant 2017;26:821-40.

6. Uchida N, Kushida Y, Kitada M, Wakao S, Kumagai N, Kuroda Y, et al. Beneficial effects of systemically administered human muse cells in adriamycin nephropathy. J Am Soc Nephrol 2017;28:2946-60.

7. Perone MJ, Gimeno ML, Fuertes F. Immunomodulatory properties and potential therapeutic benefits of muse cells administration in diabetes. In: Dezawa M, editor. MUSE Cells Endogenous Reparative Pluripotent Stem Cells. Tokyo, Japan: Springer; 2018. p. 115-29.

8. Minatoguchi S, Mikami A, Tanaka T, Minatoguchi S, Yamada Y. Acute myocardial infarction, cardioprotection, and muse cells. In: Dezawa M, editor. Muse Cells Endogenous Reparative Pluripotent Stem Cells. Tokyo,
Japan: Springer; 2018. p. 153-66.

9. Niizuma K, Borlongan CV, Tominaga T. Application of muse cell therapy to stroke. In: Dezawa M, editor. MUSE Cells Endogenous Reparative Pluripotent Stem Cells. Tokyo, Japan: Springer; 2018. p. 167-86.

10. Uchida A, Sakata H, Fujimura M, Niizuma K, Kushida Y, Dezawa M, et al. Experimental model of small subcortical infarcts in mice with longlasting functional disabilities. Brain Res 2015;1629:318-28.

11. Uchida H, Morita T, Niizuma K, Kushida Y, Kuroda Y, Wakao S, et al. Transplantation of unique subpopulation of fibroblasts, muse cells, ameliorates experimental stroke possible via robust neuronal differentiation. Stem Cells 2016;34:160-73.

12. Uchida H, Niizuma K, Kushida Y, Wakao S, Tominaga T, Borlongan CV, et al. Human muse cells reconstruct neuronal circuitry in subacute lacunar stroke model. Stroke 2017;48:428-35.

13. Uchida N, Kumagai N, Kondo Y. Application of muse cells therapy for kidney disease. In: Dezawa M, editor. MUSE Cells Endogenous Reparative Pluripotent Stem Cells. Tokyo, Japan: Springer; 2018. p. 199218.

14. Nishizuka SS, Suzuki Y, Katagiri H, Takikawa Y. Liver regeneration supported by muse cells. In: Dezawa M, editor. Muse Cells Endogenous Reparative Pluripotent Stem Cells. Tokyo, Japan: Springer; 2018. p. 21941.

15. Yabuki H, Wakao S, Kushida Y, Dezawa M, Okada Y. Human multilineage-differentiating stress-enduring cells exert pleiotropic effects to ameliorate acute lung ischemia-reperfusion injury in a rat model. Cell Transplant 2018;1:963689718761657.

16. Yabuki $H$, Watanabe $T$, Oishi $H$, Katahira M, Kanehira M, Okada Y. 
MUSE cells and ischemia-reperfusion lung injury. In: Dezawa M, editor. MUSE Cells Endogenous Reparative Pluripotent Stem Cells. Tokyo, Japan: Springer; 2018. p. 293-303.

17. Yamashita T, Kushida Y, Wakao S, Tadokoro K, Nomura E, Omote Y, et al. Therapeutic benefit of Muse cells in a mouse model of amyotrophic lateral sclerosis. Sci Rep 2020;10(1):17102.

18. Toyoda E, Sato M, Takahashi T, Maehara M, Nakamura Y, Mitani G, et al. Multilineage-differentiating stress-enduring (Muse)-like cells exist in synovial tissue. Regen Ther 2019;10:17-26.

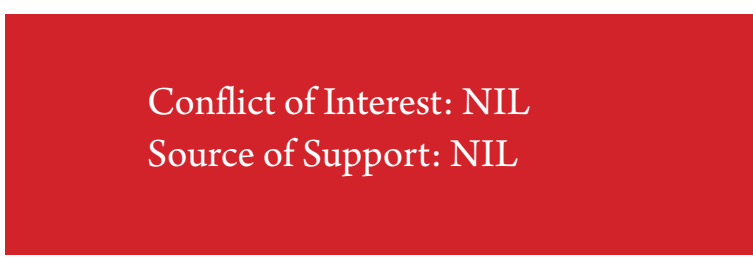

\section{How to Cite this Article}

Geli EA, Laiz P, Aniorte AF, Seijas R, Barastegui D, Díaz PÁ, Cuscó X, Sánchez C, García L, Balletbó MG, Ramón Cugat R | Multilineage-differentiating Stressenduring (MUSE) Cells in Orthobiologics: Are they the Future? I Journal of Regenerative Science | December 2021; 1(1):44-47. 